\title{
Capacidad de carga turística de las playas del Partido de Mar Chiquita, Provincia de Buenos Aires, Argentina
}

\section{Tourism carrying capacity of Mar Chiquita beaches, Buenos Aires, Argentina}

\author{
Juan M. Fernández ${ }^{l^{*}}$ y Germán R. Bértola ${ }^{2}$
}

\begin{abstract}
RESUMEN
La zona costera del Partido de Mar Chiquita presenta playas cuya geomorfología y nivel de antropización es diversa. De sur a norte, incluye los barrios costeros de Playa Dorada, Santa Elena, Santa Clara del Mar, Camet Norte, La Caleta, Mar de Cobo y Balneario Parque Mar Chiquita. Es uno de los sectores de la provincia de Buenos Aires más afectados por los procesos de erosión costera. Esta erosión es originada principalmente por tormentas sudestadas y por la incesante modificación del paisaje ribereño causada por la urbanización y las obras de defensa costera. Planificar la capacidad máxima soportable de un determinado espacio, en conjunto con una ordenación ambiental del territorio, contribuye a que dicho fenómeno no se vea incrementado. El presente trabajo se enfocó en determinar la Capacidad de Carga Turística (CCT) para las playas del Partido de Mar Chiquita, mediante el empleo de fotografías aéreas e imágenes satelitales, con el fin de determinar la carga máxima de personas que podrán acceder a dicho recurso sin representar un detrimento de la calidad de este y también analizar la evolución de la gestión municipal en relación con el manejo del turismo costero. La CCT para el área de estudio arrojó un valor de 1887.07 visitantes/día para el año 2011, comparado con los 943.55 que se obtuvieron para el año 1955.
\end{abstract}

Palabras claves: Capacidad de Carga Turística, erosión, playa, desarrollo sustentable, Partido de Mar Chiquita.

\begin{abstract}
Mar Chiquita beaches' geomorphology and anthropization is diverse. From south to north, this area includes the following coastal areas: Playa Dorada, Santa Elena, Santa Clara del Mar, Camet Norte, La Caleta, Mar de Cobo and Balneario Parque Mar Chiquita. This section is one of the most affected areas by erosion in the Buenos Aires Province. Erosion here is caused mainly by southerly storms and constant changes in the coastal landscape resulting from urbanization and the coastal defense work. Planning the maximum tolerable capacity for a given space and environmental land management help this phenomenon not to increase. This study was focused on determining the Tourism Carrying Capacity (TCC) for the Mar Chiquita beaches by using aerial photographs and satellite images in order to determinate the maximum amount of visitors in this resource without affecting its quality and analyze the evolution of municipal management in relation to coastal tourism management. TCC for the studied area was stated at 1,887.07 visitors/day in 2011, compared to 943.55 visitors for 1955 .
\end{abstract}

Keywords: Tourism Carrying Capacity, erosion, beach, sustainable development, Mar Chiquita area.

CIC e IIMyC - Grupo de Geología de Costas y Paleoecología (Univ. Nac. de Mar del Plata). E-mail: fernandezjuanmf@gmail.com* CONICET e IIMyC - Grupo de Geología de Costas y Paleoecología (Univ. Nac. de Mar del Plata).

Recibido: 26 de noviembre de 2013

Corregido: 4 de junio de 2014

Aceptado: 5 de julio de 2014

DOI: http://dx.doi.org/10.15359/revmar.6.4

Rev. Mar. Cost. ISSN 1659-455X. Vol. 6: 55-73, Diciembre 2014. 


\section{INTRODUCCIÓN}

El sector litoral de la provincia de Buenos Aires está caracterizado por diferentes ámbitos geomórficos que dependen de un conjunto de variables, no solamente relacionados con los procesos actuales, sino también con su historia evolutiva. La litología, las variaciones del nivel del mar y, más recientemente, las actividades humanas le imprimen particularidades específicas. El conjunto de estos factores determina la variabilidad de las playas (Schnack et al. 2007).

El Partido de Mar Chiquita cuenta con $67 \mathrm{~km}$ de playa. La zona estudiada (Fig. 1), comprendida en el sector con balnearios y por ello, turísticamente activa, se extiende a lo largo de $19 \mathrm{~km}$ de costa con diferentes morfologías y dinámicas. La topografía de estas es muy variada, siendo extensas y con médanos en la zona norte y más cortas y con acantilados a medida que se avanza hacia el sur. Comprende playas de bolsillo entre cabos rocosos con escasos sectores de acumulación en Playa Dorada y Santa Elena, playas de escasos metros generadas entre defensas costeras en Mar de Cobo y Mar Chiquita y playas entre espigones y cabos rocosos en Santa Clara, Camet Norte y La Caleta. De esos 19 $\mathrm{km}$, solo un $40 \%$ corresponde a playas aprovechables para el uso como recurso turístico, principalmente por su morfología y por la disposición de las defensas costeras a lo largo de la costa. Dicha situación, en sinergia con los procesos erosivos que tienen lugar en el paisaje costero, traen consigo un resultado que requiere de la máxima atención por parte del municipio y el vasto conocimiento de cada playa en particular.

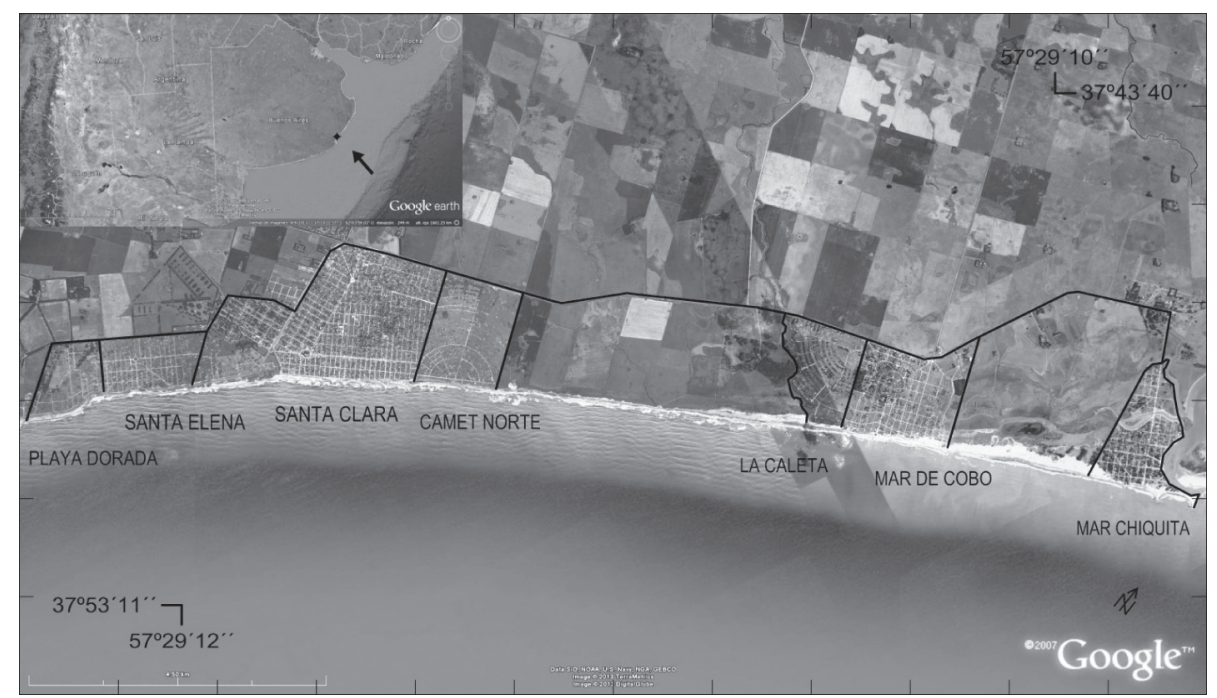

Fig. 1. Imagen satelital (28/10/2011: Spot 5: Hora: 13:29:45 UTC; Lat/Long (centro): -37.8372/-57.7359; Escala: 2.5 M COLOR) de la zona de estudio con sus límites marcados Fuente: Google Earth (1991) y retocada por el autor mediante el software Surfer 10 (2012) Fig. 1. Satellite image (10/28/2011: Spot 5: Hour: 13:29:45 UTC; Lat/Long (center): -37.8372/57.7359; Scale: 2.5 M COLOR) of study area with boundaries marked Source: Google Earth (1991) and retouched by the author using Surfer 10 (2012) 
La Capacidad de Carga (CC) es un concepto tomado por numerosas disciplinas y con significaciones diferentes según el objetivo. La definición del término que hace la Organización Mundial del Turismo (Echamendi Lorente, 2001) contempla diversos puntos de vista: "El concepto de capacidad de carga trata de establecer en términos mensurables el número de visitantes y el grado de desarrollo que es susceptible de alcanzarse sin que se produzcan situaciones perjudiciales para los recursos (...). Es la capacidad que se puede alcanzar sin daño físico para el medio natural y para el medio artificial, sin daño social/económico para la comunidad y para la cultura local o sin perjudicar el justo equilibrio entre desarrollo y conservación. Es el número de visitantes que pueden darse en un lugar a cualquier hora o durante un año sin que resulte en una pérdida de atracción o en daños para el medio ambiente. Se puede decir, en términos generales, que existe una saturación o un sobrepaso de la capacidad de carga cuando los movimientos de las personas, nacionales o internacionales, excede temporalmente el nivel aceptable por el medio ambiente físico y humano de la zona de acogida o destino".

La identificación de límites y el respeto de estos son aspectos centrales en la concreción de un desarrollo sustentable. La planificación del turismo sustentable ha propuesto el concepto de Capacidad de Carga Turística (CCT), que básicamente puede entenderse como el nivel en el cual un área, ecosistema, hábitat, destino, si- tio o paisaje puede acomodar turistas o visitantes sin llegar al punto de declinación inaceptable o irreversible de dicho sitio (Inskeep, 1991). Cifuentes (1992) hace alusión a este concepto en sus trabajos dedicados específicamente a áreas protegidas y propone que la CCT se define como el número máximo de visitas que puede recibir un área protegida con base en las condiciones físicas, biológicas y de manejo que se presentan en el área en el momento del estudio. Por lo anterior, se propone que la determinación de la CCT constituye una herramienta de planificación que permite obtener una aproximación a la intensidad de uso de las áreas destinadas al uso público, por lo que sustenta y requiere decisiones de manejo.

El objetivo se circunscribió a la CCT específicamente en playas. Además, se hizo hincapié en la Capacidad de Carga Turística de aquellas playas del Partido de Mar Chiquita que pueden ser aprovechadas para un uso turístico. Por lo anterior, el trabajo no trasciende más allá de solo cálculos, sino que se propone que los resultados sirvan como recomendaciones futuras y propuestas de gestión para el municipio administrador de las playas. A dicho concepto hacen referencia autores como Roig (2002; 2003), Betancourt Fernández y Herrera Moreno (2005), Aranguren et al. (2008) y Zacarias (2013) en artículos donde aplican dicho concepto específicamente a espacios litorales. También Salinas (2002) aclara que la CCT de una playa se debe evaluar a partir de sus condiciones geomorfológicas, ambientales, económicas y sociales, y re- 
presenta el número de visitas que puede recibir dicha playa, sin que esas variables se vean afectadas. Otros factores influyentes son la distancia a centros poblacionales, la calidad y amplitud de la franja de arena, las actividades recreativas a desarrollar, la zonificación, las infraestructuras para el servicio, la comunicación, la capacitación y el espacio mínimo necesario ocupado por un usuario (Silva, 2002).

Aquí adoptaremos la definición de Salinas (2002), ya que es la que más se asemeja a los objetivos del presente trabajo. Es necesario resaltar que se tomará en cuenta el aporte de cada uno de los trabajos relacionados con la temática (Fig. 2), con el objeto de lograr un método lo más acertado posible.

Por lo dicho, surge que determinar la CCT resulta de vital importancia para dar a conocer la capacidad óptima y máxima a la que se deberán ajustar los futuros planes e iniciativas de desarrollo urbano y turístico en zonas costeras.

Cabe destacar que el concepto abordado en el presente trabajo es por demás controversial. Esto basado en que, aunque existe un cierto consenso entre los expertos a la hora de definir la CCT, no hay aún un acuerdo sobre los métodos de medición y cuantificación a emplear ni mucho menos sobre los umbrales de permisibilidad que se consideran adecuados en cada caso. Estas dificultades de medición provienen también del interés de considerar simultáneamente parámetros físicos y ecológicos, sociales y económicos, psicológicos, antropológicos y culturales, es decir, variables mensurables y otras puramente subjetivas y no solo en el espacio receptor, sino también en las

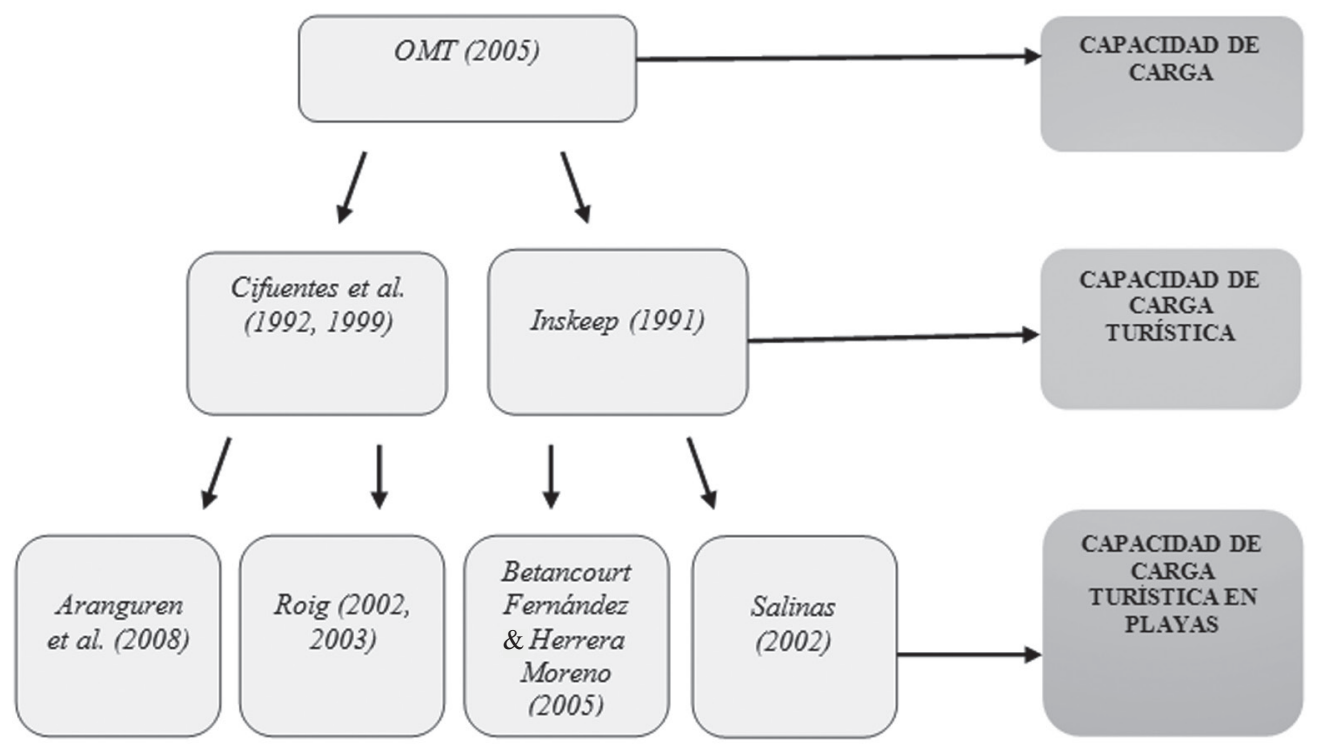

Fig. 2. Conceptualizaciones sobre CC, CCT y CCT en playas, de los diferentes autores, contempladas en el presente estudio

Fig. 2. Conceptualizations of CC, CCT and CCT on beaches from different authors used in this study 
zonas emisoras e intermedias o de tránsito (Cazes, 1992).

La determinación de la CCT no debe ser tomada como una solución a los problemas de visitación de un recurso natural. Es tan solo una herramienta de planificación que sustenta decisiones $\mathrm{y}$ acciones de manejo. En fin, para ser asertivos en el manejo sostenible de un espacio de interés turístico se requieren diversas estrategias, entre las cuales la CCT es una de ellas. Dicha estimación debe estar apoyada por diseños de ingeniería, manejo de aguas servidas, manejo de desechos sólidos, reglamentos de uso, zonificaciones de uso, medidas económicas, de vigilancia y control, así como servicios de diversa índole. Así, debe quedar claro que la CCT resulta un complemento de las estrategias de gestión.

\section{METODOLOGÍA}

Para analizar la CCT de las playas del Partido de Mar Chiquita se emplearon fotografías aéreas a escala 1:10000 de los años 1955 (Servicio de Hidrografía Naval-División Fotogramétrica-Armada Argentina), de los años 1967 (Laboratorio de Ensayo de Materiales-Sección Geología) y 1987 (Geodesia), imágenes Digital Globe de los años 2003 y de los años 2009 (03/05/2003: Digital Globe: Catalog ID: 1010010001DDAF03; Cloud Cover: 0\%; Quality: 90. 03/05/2003: Digital Globe: Catalog ID: 1010010001DDAF02; Cloud Cover: 0\%; Quality: 90. 15/03/2009: Digital Globe: Catalog ID: 10100100094F3101; Cloud Cover: 25\%; Quality: 99) e imágenes SPOT del año 2011 (28/10/2011:
Spot 5: Hour: 13:29:45 UTC; Lat/Long (center): -37.8372/-57.7359; Scale: 2.5 M COLOR. 06/08/2011: Spot 5: Hour: 13:27:03 UTC; Lat/Long (center): -37.8369/-57.0174; Scale: 2.5 M COLOR), provenientes de Google Earth (1991). La metodología de cálculo de la CCT fue la de Cifuentes et al. (1999) con modificaciones adosadas por los autores.

Se realizó un análisis detallado de cada sección de playa, para cada período, mediante el software Image J (2007), cuya prestación permite realizar mediciones de alta precisión en cualquier tipo de imagen. Se procedió, en primera instancia, a recorrer el área de estudio con el motivo de identificar la morfología de la costa utilizando GPS, para de esta manera almacenar puntos y rutas de interés para luego ingresarlos al software antes mencionado como medidas conocidas. Hecho este procedimiento, se individualizaron las diferentes playas en cada foto e imagen, para determinar la superficie de playa tanto pública como privada. Cabe destacar que para el análisis de la CCT solo se tomó en cuenta el área correspondiente al dominio público. Para los objetivos del presente artículo, entenderemos por playa, según Codignotto (1987), la zona de material no consolidado que se extiende hacia tierra desde la línea de baja marea hasta donde se nota un cambio notable de los materiales o en las condiciones fisiográficas, o donde la línea de vegetación es permanente. Por dominio público de la playa entendemos aquella superficie no ocupada por concesiones de balnearios 
o alguna otra obra de infraestructura privada (ver zonificación).

Con el objeto de recabar información imposible de visualizar a través de fotografías aéreas e imágenes satelitales se realizaron salidas de campo y encuestas, tanto a dueños de balnearios como a pobladores locales.

El proceso de obtención de los valores de la CCT consta del cálculo basado en tres niveles: Cálculo de Capacidad de Carga Física (CCF), Cálculo de Capacidad de Carga Real (CCR) y Cálculo de Capacidad de Carga Efectiva (CCE). Estos niveles tienen una relación que se representa de la siguiente manera:

\section{$C C F>C C R>C C E$}

\section{Capacidad de Carga Física}

"Es el límite máximo de visitas que se pueden hacer al sitio durante el día. Está dada por la relación entre factores de visita (horario y tiempo de visita), el espacio disponible y la necesidad de espacio por visitante" (Cifuentes et al. 1999) y se define como:

$$
C C F=\left(S / S P^{*} N V\right)
$$

\section{Donde:}

$\mathrm{S}$ : es la superficie disponible en metros cuadrados para cada playa. SP: es la superficie promedio en metros cuadrados usada por una persona.

$\mathrm{NV}$ : es el número de veces que el sitio puede ser visitado por una misma persona en un día; se determina con la fórmula: $N V=H v / T v$.

Hv: es el horario de visita.

Tv: es el tiempo promedio de visita.
Se procedió a calcular la CCT para cada una de las playas analizadas del Partido de Mar Chiquita (Playa Dorada, Santa Elena, Santa Clara del Mar, Camet Norte, La Caleta, Mar de Cobo, Balneario Parque Mar Chiquita) para los años 1955, 1967, 1987, 2003, 2009 y 2011. La cifra obtenida en el cálculo anterior representa la CCF del destino, pero no es la capacidad real, ya que para obtener esta última se necesitan realizar correcciones propias de cada sitio que permiten llegar a un valor ajustado a dichas condiciones (Cifuentes et al. 1999; Segrado \& Arroyo, 2009). De este modo, la CCF se reduce a una CCR por la combinación de criterios ecológicos y antropológicos, que se denominan factores de corrección. Estos factores son "ajustes limitantes" que incorporan ciertas restricciones físicas a la CCR.

\section{Capacidad de Carga Real}

Es el máximo de visitas de un sitio determinado, luego de someter la CCF a los factores de corrección nombrados por Cifuentes et al. (1999). Se obtienen considerando variables físicas, ecológicas y sociales, que pueden ser distintas para cada destino o sitio turístico. Actualmente, la elección de estas variables o factores de corrección se establecen con criterios cualitativos, por lo que solo puede ser realizada por expertos conocedores del destino analizado, luego de identificar las "limitantes críticas" a las que se refieren Cifuentes et al. (1999) (op. cit.).

La CCR se establece con la siguiente fórmula: 
$\mathrm{CCR}=\mathrm{CCF}(\mathrm{FC} 1 \times \mathrm{FC} 2 \times \mathrm{FC} 3 \mathrm{x}$ FC4 x FC5 x...x FCn)

Donde los factores de corrección (FC) se expresan en porcentaje o probabilidad que se descuenta de la CCF. Para calcularlos se usa la fórmula general:

$$
F C=1-(M l / M t)
$$

Donde:

FC: es el factor de corrección.

Ml: es la magnitud limitante.

Mt: es la magnitud total.

Ninguna investigación puede abarcar la totalidad de las relaciones o fenómenos de un sistema, por lo que siempre será necesario aplicar criterios de selección. Como comentara Becerra (1997): "Dejar elementos o relaciones fuera del sistema o modelo construido no significa ignorarlos. Se trata de una división metodológica ineludible".

Los factores de corrección adoptados en este trabajo fueron los siguientes:

- Factor de precipitación (FCpp): se calcularon las precipitaciones por mes para los meses de verano (de diciembre a marzo) y luego se realizó la sumatoria de estas. Los datos fueron obtenidos a partir de estudios de_Mundomanz (2013), sitio web que proporciona datos meteorológicos obtenidos de los mensajes SYNOP emitidos por estaciones meteorológicas oficiales, así como datos meteorológicos previstos basados en los modelos de predicción globales GFS y ECMWF.
Se obtuvo un total de $790 \mathrm{~mm} /$ año para el área de estudio, de los cuales $379.8 \mathrm{~mm}$ se producen en los meses de verano.

- Factor de brillo solar (FCbsol): se calcularon las horas de sol limitantes para cada uno de los 121 días de los meses de verano. Se tomaron como limitantes las horas de sol de máxima intensidad, siendo estas de $5 \mathrm{hs}$, comprendidas entre las 11:00 y las 16:00 hs. Estas últimas se relacionaron con el total de 12 horas de sol para los meses de verano, comprendidas entre las 8:00 y las 20:00 hs.

- Factor de sudestadas (FCsud): según García (2011), las sudestadas son más notorias en invierno y primavera, aunque no se descartan también durante el verano. Es un estado de mal tiempo que afecta al río de la Plata y al litoral de la provincia de Buenos Aires. Se caracteriza por la ocurrencia de vientos de regulares a fuertes, con velocidades mayores a $35 \mathrm{~km} / \mathrm{h}$ del sector sudeste, con precipitaciones persistentes y temperaturas relativamente bajas. Su duración promedio es de dos días. Se calculó la cantidad de sudestadas que se producen durante los meses de verano con base en García (2011), quien analizó un total de 84 sudestadas entre 1971 y el 2007. El autor menciona que se producen un promedio de 2.2 sudestadas por año, siendo los meses comprendidos entre abril 
y octubre aquellos cuyas probabilidades son mayores, rondando el $65 \%$ de probabilidad. El otro $35 \%$ correspondería a los meses de verano. Según este último dato, se calculó la cantidad de sudestadas promedio para los meses de verano, siendo este último un valor de 0.77 .

- Factor de tormentas (FCtorm): según García (2011), las tormentas se caracterizan por una situación en la que el aire más cálido y húmedo se eleva, condensa su humedad y forma cumulunimbus, que luego se precipitan. Se calculó la cantidad de tormentas que se producen durante los meses de verano con base en García (2011), quien analizó un total de 333 tormentas entre 1971 y el 2007. García (2011) menciona que se producen un promedio de 5.4 tormentas por año, siendo los meses de verano aquellos cuyas probabilidades son mayores y rondan el $60 \%$. Según este último dato se calculó la cantidad de tormentas promedio para los meses de verano, siendo este último un valor de 3.24. Entonces, si un $60 \%$ de tormentas se producen en verano, de las 5.4 tormentas que se producen en promedio por año, entonces, 3.24 tormentas se circunscriben a los meses de diciembre a marzo.

- Factor de erosión (FCero): Se determinaron los metros de playa susceptibles de ser erosionados con base en las tasas de erosión. Esto arrojó un valor de $10500 \mathrm{~m}$ de costa erosionables de un total de $19000 \mathrm{~m}$ de costa del área de estudio. Sin embargo, la veracidad de los factores de corrección está dada por aquellos factores que realmente son "limitantes". Por tal motivo, solo se consideran $1700 \mathrm{~m}$ correspondientes al Balneario Parque Mar Chiquita, cuya susceptibilidad es la más alta, dada su elevada tasa de erosión (7 m/año, Isla, 1997).

Luego de obtener el factor de corrección (FC) de cada indicador, se puede determinar cuál es la Capacidad de Carga Real (CCR) del destino turístico. Según el método de Cifuentes et al.(1999), se deben descontar todos los indicadores arriba analizados a la CCF. Entonces:

$$
\begin{aligned}
& C C R=C C F(\text { FCpp } x \text { FCbsol } x \\
& \text { FCsud } x \text { FCtorm } x \text { FCero })
\end{aligned}
$$

Capacidad de Carga Efectiva, Permisible o de Acogida

En la última fase, la Capacidad de Carga Efectiva (CCE) representa el número máximo de visitas que se puede permitir en un lugar determinado diariamente (tomando en cuenta la capacidad de gestión por parte de los administradores de playa y los servicios prestados al visitante). La CCE se determina con esta fórmula:

$$
C C E=C C R \times C G \text {. }
$$


Donde:

CCR: es la capacidad de Carga Real. CG: es la capacidad de Gestión.

La Capacidad de Gestión (CG) es el límite económico y social que tiene el sector privado y/o el municipio para la gestión de sus actividades y el logro de objetivos. Aquí intervienen factores tales como: normas jurídicas, políticas y acciones, equipamiento específico del sector turístico, personal técnico y operativo, financiamiento, infraestructura y facilidades o instalaciones disponibles (Cifuentes, 1992). Las variables de cada factor seleccionado serán valoradas por su presencia o ausencia. A la presencia del total de variables de cada factor se le otorgará el valor de 1. Cada variable ausente se descontará según la cantidad de ítems que cada factor tenga y qué porcentaje represente de este. Se presenta un total de 23 ítems seleccionados y analizados. Para lograr una estimación objetiva de la $C G$ es fundamental uniformar el mecanismo de calificación para todas las variables (Cifuentes, 1992). Los factores utilizados fueron:

1. Infraestructura: (10 items):

Pública:casetas deguardavidas, baños, iluminación, servicio de información.

Privada: construcción balnearia, baños con vestuarios, proveeduría, carpas y sombrillas, señalización, facilidad de acceso.

2. Equipamiento: (7 items):

Cestos de basura, quiosco, bar, restaurante, solárium, piscina, espacios para realizar actividades recreativas.

\section{Personal: (3 items):}

Capacitación ambiental, dotación adecuada, jornada laboral coherente ( 8 hs por turno).

4. Respaldo jurídico: (2 items):

En regla con respecto a planes integrales costeros, normativa de construcciones balnearias.

La definición de la $\mathrm{CG}$ no fue sencilla de obtener, ya que a nivel público, los datos sobre las variables definidas no son de fácil acceso o presentan situaciones disímiles.

Los cálculos para el área de estudio se basaron en los siguientes supuestos:

- Se establece que una persona requiere para su desplazamiento de $1 \mathrm{~m}^{2}$ de espacio. El espacio estándar internacional para realizar actividades recreativas en espacios públicos es de 4 $\mathrm{m}^{2} /$ usuario (OMT, 2005). Sin embargo, existe una superficie óptima, en relación con el espacio, para destinos turísticos estacionales y es de $15 \mathrm{~m}^{2} /$ usuario (Roig, 2002).

- La mayoría de los usuarios de playa se ubican a menos de $50 \mathrm{~m}$ del mar y por encima de la línea de marea alta (Silva, 2002). Se ha observado en otros estudios que a partir del límite de esta franja de $50 \mathrm{~m}$, la densidad de usuarios desciende de forma muy rápida.

- Paralos cálculosdelaCCT, solose consideró el área correspondiente al dominio público de cada playa y para cada año. 
- La playa es aprovechable solo para los meses de verano (de diciembre a marzo) y considerando el horario de sol (de 8:00 a 20:00 hs) en relación con el horario de máxima afluencia al destino. Se consideró un máximo promedio de 6 hs por día que el visitante permanece en la playa.

\section{Zonificación}

La zonificación de la playa en el momento de realizar un análisis de la CCT es sumamente determinante, dado que no todas las áreas de la playa son ocupadas o usadas con la misma frecuencia e intensidad. Por tal motivo, para el análisis de la CCT en las playas del Partido de Mar Chiquita, la medición se circunscribió solo al área correspondiente al dominio público (exposición solar + circulación), con una propuesta de zonificación modificada de Betancourt Fernández \& Herrera Moreno (2005) y adaptada a la tipología de playas en cuestión (Fig. 3):

- Área de unidades de sombra: franja más lejana a la línea de costa, detrás de la zona de exposición solar. Su dimensión depende del ancho de la playa, de la cantidad de metros destinados a la infraestructura balnearia y de las unidades de sombra que la licitación incluya. Actividades: ingreso/egreso y búsqueda de un espacio en la playa. Aquí se ubican los centros de servicio y se alternan actividades recreativas y de descanso al sol y la sombra dentro de los límites de la infraestructura balnearia.

- Área de exposición solar: franja de arena por encima del límite de marea alta (transición arena seca/arena húmeda), variable según las características geomorfológicas de la playa. Su longitud y ancho determinan la capacidad de carga física máxima de la playa. Actividades: los usuarios descansan al sol y aprovechan elementos naturales (vegetación) o artificiales (sombrillas) para darse sombra. Se realizan actividades recreativas, deportivas $\mathrm{y} / \mathrm{o}$ de descanso que no requieren de instalaciones permanentes.

- Área de circulación: franja de playa influida por las mareas, en constante contacto con el mar $\mathrm{y}$ que posee firmeza al paso. $\mathrm{Su}$ ancho varía según la pendiente de la playa y la amplitud de la marea. Actividades: entrada y salida de bañistas, caminatas $y$ se desarrollan diversas actividades de recreación.

- Para llevar a cabo dicha zonificación se procedió, en primera instancia, a la captura y procesamiento de imágenes satelitales provenientes de Google Earth (1991) del año 2011 (Fig. 4a), para luego someterlas al software Corel Photo-Paint X5 (2008), permitiendo un análisis e identificación claros de los diferentes límites de interés del trabajo, gracias a su herramien- 


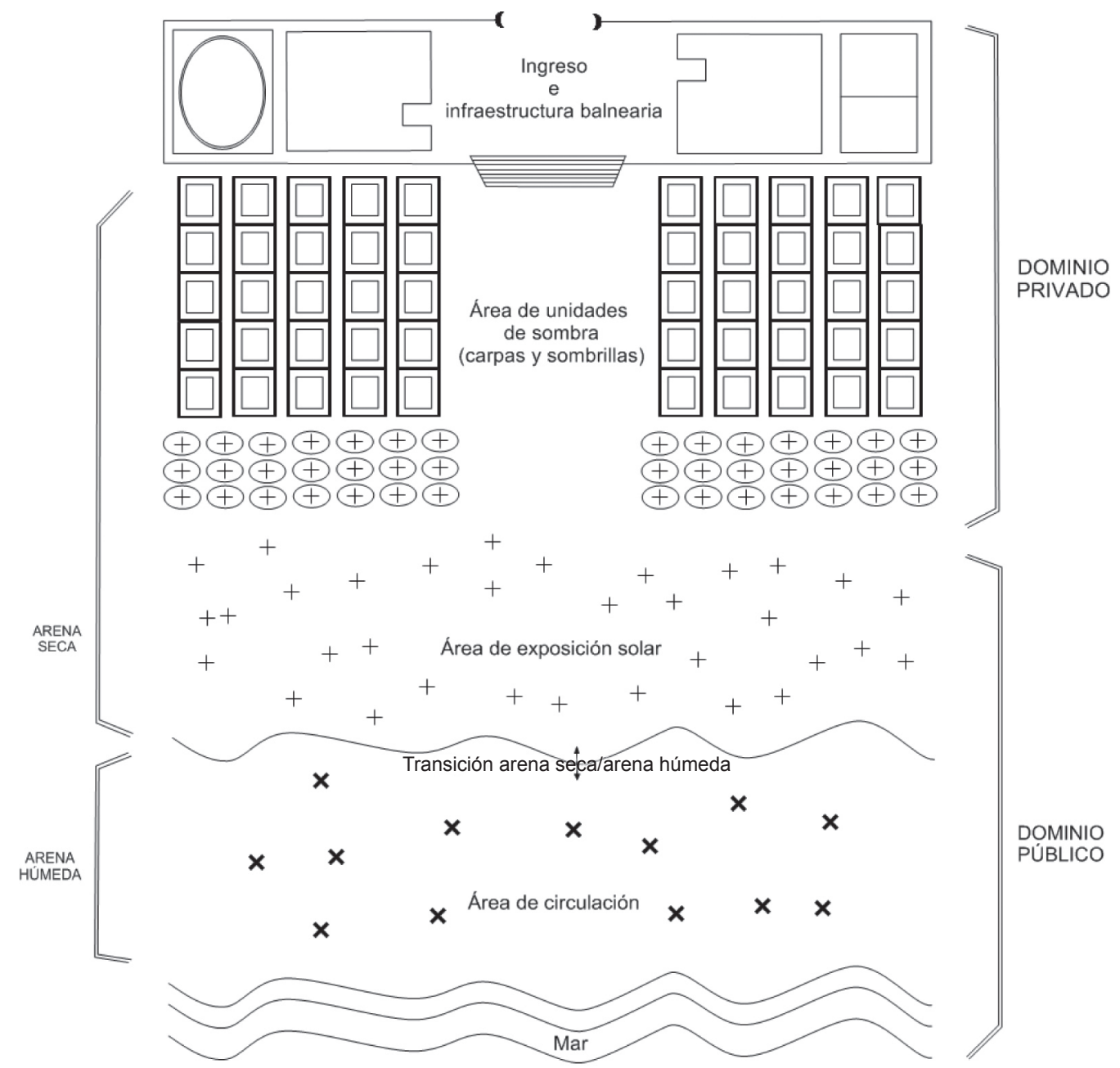

Fig. 3. Esquema de una playa modelo zonificada

Fig. 3. Outline of a model zoned beach

ta de detección de bordes (Fig. 4b). Luego, mediante el software Surfer 10 (2012), se llevó a cabo la georreferenciación de las imágenes, así como la marcación de los límites antes mencionados de nuestra zonificación (Fig. 4c). La zonificación propuesta se presenta en la Fig. 4, y fue adaptada a una playa correspondiente al Balneario Costa Corvinas (Fig.
4) de la localidad de Santa Clara del Mar. La secuencia de imágenes propuestas a continuación ilustra lo dicho.

\section{RESULTADOS}

A continuación se presentará el cálculo de la CCF para la superficie total de playas y para el año más reciente en que se obtuvieron datos.

$\mathrm{S}$ : superficie de playa disponible en metros cuadrados: $302560.8 \mathrm{~m} 2$ 


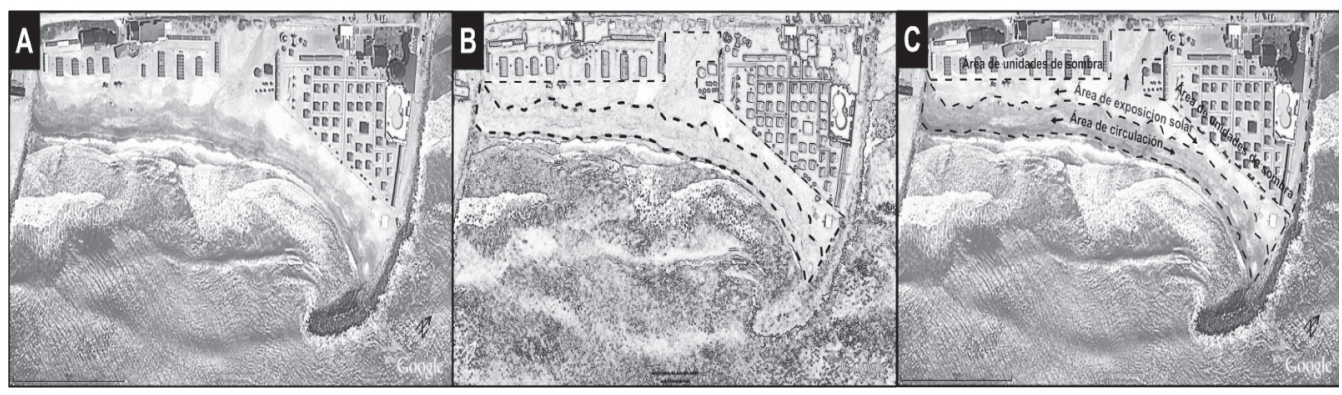

Fig. 4. Secuencia de imágenes (A, B, C) de la zona de Santa Clara del Mar descriptiva de la metodología de zonificación propuesta para la zona de estudio. Fuente: Google Earth (1991)

Fig. 4. Image sequence (A, B, C) of Santa Clara del Mar describing the zoning methodology proposed for the study area. Source: Google Earth (1991)

SP: superficie ocupada por una persona: $4 \mathrm{~m} 2$

NV: número de veces que la playa puede ser visitada/persona/día: 2 veces

Hv: horario de visitas. Verano: 8:00 a $20: 00=12$ hs.

Tv: tiempo de visita: 6 hs.

$\mathrm{NV}=12 / 6=2$

$\mathrm{CCF}=(302560.8 / 4) * 2=151$

280.4 visitantes

El paso a seguir es: calcular los factores de corrección y así se obtiene la CCR:

- Factor de precipitación (FCpp): De esta manera: 379.8 $\mathrm{mm} / 790 \mathrm{~mm}=0.48$. De este modo, FCpp $=1-0.48=0.52$.

- Factor de brillo solar (FCbsol): De esta manera: Horas de sol limitantes: 121 x $5=605$; Horas de sol en verano: $121 \mathrm{x}$ $12=1452$. De este modo, 605 / $1452=0.41$ y FCbsol $=1$ $0.41=0.584$.

- Factor de sudestadas (FCsud):
De esta manera: Número de sudestadas/verano: 0.77; Número de sudestadas/año: 2.2. De este modo, $0.77 / 2.2$ $=0.35$ y $0.35 \times 2=0.7$ ( $\mathrm{se}$ multiplica por 2 , dado que la duración promedio es de dos días), por ello se desprende que FCsud $=1-0.7=0.3$.

- Factor de tormentas (FCtorm): De esta manera: Número de tormentas en verano: 3.24; Número de tormentas en el año: 5.4. De este modo, $3.24 / 5.4=$ 0.6 y FCtorm $=1-0.6=0.4$.

- Factor de erosión (FCero): De esta manera: Metros de máxima susceptibilidad a ser erosionados: 1700 ; Metros lineales totales de playa: 19 000. De este modo, 1700 $/ 19000=0.09$ y FCero $=1$ $-0.09=0.911$.

CCR $=$ CCF (FCpp $\mathrm{x}$ FCbsol $\mathrm{x}$ FCsud x FCtorm x FCero)

$\mathrm{CCR}=151280.4(0.52 \times 0.584 \times$ 


$$
\begin{aligned}
& 0.3 \times 0.4 \times 0.911) \\
& \mathrm{CCR}=151280.4 \times 0.033 \\
& \mathrm{CCR}=4992.25 \text { visitantes }
\end{aligned}
$$

Para calcular la CCE se obtuvo en primera instancia la $\mathrm{CG}$, mediante la siguiente puntuación de factores (aquí se presenta a modo de guisa para el año 2011). Como se aprecia en el cuadro 1, sobre un total de 23 ítems buscados, en Playa Dorada se encontraron 10. Para Santa Elena se obtuvieron 11. En Santa Clara del Mar se relevaron 17 ítems presentes. Para Camet Norte se localizaron 3. En La Caleta se hallaron 3, 6 en Mar de Cobo y para el Balneario Parque Mar Chiquita se encontraron 11.

\section{Puntuación de factores}

1. Playa Dorada: $10 / 23=0.434$.

2. Santa Elena: $11 / 23=0.478$.

Cuadro 1. Ítems a considerar (con sus respectivas referencias) para el cálculo de la

\begin{tabular}{|c|c|c|c|c|c|c|c|c|c|c|c|c|c|c|c|c|c|c|c|c|c|c|c|}
\hline \multirow{3}{*}{ Playa } & \multicolumn{11}{|c|}{ Infraestructura } & \multirow{2}{*}{\multicolumn{6}{|c|}{ Equipamiento }} & \multirow{2}{*}{\multicolumn{3}{|c|}{ Personal }} & \multirow{2}{*}{\multicolumn{2}{|c|}{$\begin{array}{c}\text { Respaldo } \\
\text { jurídico }\end{array}$}} & \multirow{3}{*}{ Total } \\
\hline & \multicolumn{5}{|c|}{ Pública } & \multicolumn{6}{|c|}{ Privada } & & & & & & & & & & & & \\
\hline & $\mathbf{A}$ & B & $\mathbf{C}$ & D & $\mathbf{E}$ & $\mathbf{F}$ & G & $\mathbf{H}$ & $\mathbf{I}$ & $\mathbf{J}$ & $\mathbf{K}$ & $\mathbf{L}$ & $\mathbf{M}$ & $\mathbf{N}$ & $\mathbf{O}$ & $\mathbf{P}$ & $\mathbf{Q}$ & $\mathbf{R}$ & $\mathbf{S}$ & $\mathbf{T}$ & $\mathbf{U}$ & $\mathbf{V}$ & \\
\hline PD & $\mathrm{x}$ & & & & $\mathrm{x}$ & $\mathrm{x}$ & & $\mathrm{x}$ & $\mathrm{x}$ & & $\mathrm{x}$ & $\mathrm{x}$ & & $\mathrm{x}$ & & $\mathrm{x}$ & & & $\mathrm{x}$ & & & & 10 \\
\hline SE & $\mathrm{x}$ & & & & $\mathrm{x}$ & $\mathrm{x}$ & & $\mathrm{x}$ & $\mathrm{x}$ & & $\mathrm{x}$ & $\mathrm{x}$ & & $\mathrm{x}$ & $\mathrm{x}$ & $\mathrm{x}$ & & & $\mathrm{x}$ & & & & 11 \\
\hline $\mathrm{SC}$ & $\mathrm{x}$ & $\mathrm{x}$ & & $\mathrm{x}$ & $\mathrm{x}$ & $\mathrm{x}$ & $\mathrm{x}$ & $\mathrm{x}$ & $\mathrm{x}$ & $\mathrm{x}$ & $\mathrm{x}$ & $\mathrm{x}$ & $\mathrm{x}$ & $\mathrm{x}$ & $\mathrm{x}$ & $\mathrm{x}$ & $\mathrm{X}$ & & $\mathrm{x}$ & & & & 17 \\
\hline $\mathrm{CN}$ & $\mathrm{x}$ & & & & & & & & & & & & & & & & & & & & $\mathrm{x}$ & $\mathrm{X}$ & 3 \\
\hline LC & $\mathrm{x}$ & & & & & & & & & & & & & & & & & & & & $\mathrm{x}$ & $\mathrm{X}$ & 3 \\
\hline MCO & $\mathrm{x}$ & & & & & & & $\mathrm{x}$ & & & & & $\mathrm{x}$ & & & & & & $\mathrm{x}$ & & $\mathrm{x}$ & $\mathrm{x}$ & 6 \\
\hline МСН & $\mathrm{x}$ & & & & $\mathrm{x}$ & $\mathrm{X}$ & $\mathrm{X}$ & $\mathrm{X}$ & & $\mathrm{x}$ & $\mathrm{x}$ & $\mathrm{x}$ & $\mathrm{x}$ & $\mathrm{X}$ & & & & & $\mathrm{x}$ & & & & 11 \\
\hline
\end{tabular}
Capacidad de Gestión de las playas del Partido de Mar Chiquita para el año 2011 Table 1. Items to be considered to calculate Mar Chiquita beaches management capacity for 2011

Referencias: PD: Playa Dorada; SE: Santa Elena; SC: Santa Clara del Mar; CN: Camet Norte; LC: La Caleta; MCO: Mar de Cobo; MCH: Balneario Parque Mar Chiquita; A: casetas de guardavidas; B: baños; C: iluminación; D: servicios de información; E: construcción balnearia; F: baños con vestuarios; G: proveeduría; H: carpas y sombrillas; I: señalización; J: facilidad de acceso; K: cestos de basura; L: quiosco; M: bar; N: restaurante; O: solárium; P: piscina; Q: espacios para actividades recreativas; R: capacitación ambiental; S: dotación adecuada; T: jornada laboral coherente; U: plan de manejo del espacio costero; V: normativa de construcciones balnearias.

References: PD: Playa Dorada; SE: Santa Elena; SC: Santa Clara del Mar; CN: Camet Norte; LC: La Caleta; MCO: Mar de Cobo; MCH: Balneario Parque Mar Chiquita; A: Lifeguard stands; B: restrooms; C: lighting; D: information services; E: beach resort development; F: restrooms and dressing rooms; G: convenience store; H: tents and umbrellas; I: signaling; J: easy access; K: trash cans; L: kiosk; M: bar; N: restaurant; O: solarium; P: pool; Q: spaces for recreational activities; R: environmental training; $\mathrm{S}$ : adequate distribution of tasks; $\mathrm{T}$ : optimal working hours; U: coastal management; V: rules of seaside buildings. 
Cuadro 2. Resultados del cálculo de la Capacidad de Carga Física, Física óptima, Real y Efectiva para cada playa y período

Table 2. Results of Physical Carrying Capacity, Optimal Physical Capacity, and Real and Effective Capacity for each beach and period

\begin{tabular}{|c|c|c|c|c|c|c|c|c|c|}
\hline $\begin{array}{l}\text { Playa } \\
\end{array}$ & Año & $\begin{array}{l}\text { Longitud } \\
\text { (m) }\end{array}$ & $\begin{array}{l}\text { Superficie } \\
\text { Total }\left(\mathbf{m}^{2}\right)\end{array}$ & $\begin{array}{c}\text { Superficie } \\
\text { Pública }\left(\mathrm{m}^{2}\right)\end{array}$ & $\begin{array}{c}\text { Superficie } \\
\text { Privada } \\
\left(\mathbf{m}^{2}\right)\end{array}$ & $\begin{array}{c}\mathrm{CCF} \\
\text { (pers.) }\end{array}$ & $\begin{array}{c}\text { CCF } \\
\text { óptima } \\
\text { (pers.) }\end{array}$ & $\begin{array}{c}\mathrm{CCR} \\
\text { (pers.) }\end{array}$ & $\begin{array}{c}\mathrm{CCE} \\
\text { (pers.) }\end{array}$ \\
\hline \multirow{6}{*}{ 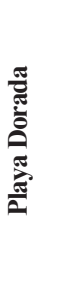 } & 1955 & 75.15 & 9326.65 & 9326.65 & 0 & 4663 & 1243 & 153.89 & 56.94 \\
\hline & 1967 & 146.2 & 2796.5 & 2796.5 & 0 & 1398 & 373 & 46.14 & 17.07 \\
\hline & 1987 & 162.5 & 3076.09 & 2656.34 & 419.75 & 1328 & 354 & 43.83 & 16.22 \\
\hline & 2003 & 219.3 & 3512.68 & 2594.47 & 918.21 & 1297 & 344 & 42.81 & 15.84 \\
\hline & 2009 & 235.46 & 9433.41 & 8394.98 & 1038.43 & 4197 & 1119 & 138.52 & 51.25 \\
\hline & 2011 & 213.75 & 5268.7 & 4036.2 & 1232.5 & 2018 & 538 & 66.60 & 24.64 \\
\hline \multirow{6}{*}{ 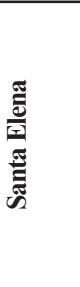 } & 1955 & 50.35 & 2611.73 & 2611.73 & 0 & 1306 & 348 & 43.09 & 15.94 \\
\hline & 1967 & 98.96 & 2281.99 & 2281.99 & 0 & 1141 & 304 & 37.65 & 13.93 \\
\hline & 1987 & 98.1 & 4376.91 & 4075.87 & 301.04 & 2038 & 542 & 67.25 & 24.88 \\
\hline & 2003 & 148.1 & 3916.63 & 3373.7 & 542.93 & 1687 & 448 & 55.67 & 20.60 \\
\hline & 2009 & 178.65 & 3650.85 & 2939.67 & 711.18 & 1470 & 390 & 48.50 & 17.95 \\
\hline & 2011 & 206.17 & 3916.16 & 3227.86 & 688.3 & 1614 & 430 & 53.26 & 19.71 \\
\hline \multirow{6}{*}{ 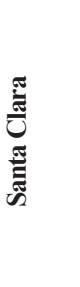 } & 1955 & - & - & - & - & - & - & - & - \\
\hline & 1967 & 2050.87 & 44996.63 & 44996.63 & 0 & 22498 & 5998 & 742.44 & 274.70 \\
\hline & 1987 & 2118.7 & 201206.58 & 196372.05 & 4834.53 & 98186 & 26182 & 3240.14 & 1198.85 \\
\hline & 2003 & 2103.2 & 94633.75 & 77594.6 & 17039.15 & 38797 & 10344 & 1280.31 & 473.72 \\
\hline & 2009 & 2126.66 & 88810.8 & 68686.4 & 20124.4 & 34343 & 9158 & 1133.33 & 419.33 \\
\hline & 2011 & 2133.84 & 176777 & 155736.79 & 21040.21 & 77868 & 20764 & 2569.66 & 950.77 \\
\hline \multirow{6}{*}{ 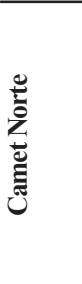 } & 1955 & - & - & - & - & - & - & - & - \\
\hline & 1967 & 1221.43 & 9957.15 & 9957.15 & 0 & 4979 & 1326 & 164.29 & 60.79 \\
\hline & 1987 & 1257.4 & 32140.5 & 32140.5 & 0 & 16070 & 4284 & 530.32 & 196.22 \\
\hline & 2003 & 1212.35 & 15846.05 & 15846.05 & 0 & 7923 & 2112 & 261.46 & 96.74 \\
\hline & 2009 & 1244.75 & 32016.75 & 32016.75 & 0 & 16008 & 4268 & 528.28 & 195.46 \\
\hline & 2011 & 1242.25 & 23214.43 & 23214.43 & 0 & 11607 & 3094 & 383.04 & 141.72 \\
\hline \multirow{6}{*}{ Uુ } & 1955 & - & - & - & - & - & - & - & - \\
\hline & 1967 & - & - & - & - & - & - & - & - \\
\hline & 1987 & 453 & 16279.15 & 16247.3 & 31.85 & 8124 & 2166 & 268.08 & 99.19 \\
\hline & 2003 & 450.6 & 12213.97 & 12074.54 & 139.43 & 6037 & 1608 & 199.23 & 73.72 \\
\hline & 2009 & 508.63 & 14747.62 & 14608.19 & 139.43 & 7304 & 1946 & 241.03 & 89.18 \\
\hline & 2011 & 527.6 & 10437.43 & 10298 & 139.43 & 5149 & 1372 & 169.92 & 62.87 \\
\hline \multirow{6}{*}{$\begin{array}{l}\frac{8}{0} \\
\dot{0} \\
\dot{0} \\
\dot{\Xi}\end{array}$} & 1955 & - & - & - & - & - & - & - & - \\
\hline & 1967 & 1623.08 & 6704.7 & 6704.7 & 0 & 3352 & 892 & 110.63 & 40.93 \\
\hline & 1987 & 1573.18 & 30119.4 & 30119.4 & 0 & 15060 & 4014 & 496.97 & 183.88 \\
\hline & 2003 & 1604.46 & 63225.24 & 63163.04 & 62.2 & 31582 & 8420 & 1042.19 & 385.61 \\
\hline & 2009 & 1611.15 & 36496.36 & 36496.36 & 0 & 18248 & 4866 & 602.19 & 222.81 \\
\hline & 2011 & 1591.92 & 42710.11 & 42710.11 & 0 & 21355 & 5694 & 704.72 & 260.75 \\
\hline
\end{tabular}


Capacidad de carga turística de las playas del Partido de Mar Chiquita, Provincia de Buenos Aires, Argentina

\begin{tabular}{cccccccccc}
\hline & $\mathbf{1 9 5 5}$ & 1884.5 & 64709.64 & 64709.64 & 0 & 32355 & 8626 & 1067.71 & 395.05 \\
& $\mathbf{1 9 6 7}$ & 2206.07 & 30051.95 & 30051.95 & 0 & 15026 & 4006 & 495.86 & 183.47 \\
& $\mathbf{1 9 8 7}$ & 1724.42 & 103690.36 & 103690.36 & 0 & 51845 & 13824 & 1710.89 & 633.03 \\
& $\mathbf{2 0 0 3}$ & 1686.73 & 44903.27 & 44468.07 & 435.2 & 22234 & 5928 & 733.72 & 271.48 \\
& $\mathbf{2 0 0 9}$ & 1704.5 & 56850.4 & 56850.4 & 0 & 28425 & 7580 & 938.03 & 347.07 \\
& $\mathbf{2 0 1 1}$ & 1697.1 & 63337.4 & 63337.4 & 0 & 31669 & 8444 & 1045.07 & 386.67 \\
\hline
\end{tabular}

Cuadro 3. Resultados del cálculo de la Capacidad de Carga Física, Física óptima, Real y Efectiva final para el área total de playa para los períodos 1955 y 2011

Table 3. Results of Physical Carrying Capacity, Optimal Physical Capacity, and Real and Effective Capacity for total study area for the periods 1955 and 2011

\begin{tabular}{|c|c|c|c|c|c|c|c|c|c|}
\hline \multirow{3}{*}{ 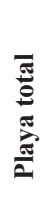 } & Año & $\begin{array}{l}\text { Longitud } \\
\text { (m) }\end{array}$ & $\begin{array}{l}\text { Superficie } \\
\text { Total }\left(\mathbf{m}^{2}\right)\end{array}$ & $\begin{array}{c}\text { Superficie } \\
\text { Pública }\left(\mathbf{m}^{2}\right)\end{array}$ & $\begin{array}{c}\text { Superficie } \\
\text { Privada } \\
\left(\mathbf{m}^{2}\right)\end{array}$ & $\begin{array}{c}\text { CCF } \\
\text { (pers.) }\end{array}$ & $\begin{array}{c}\text { CCF } \\
\text { óptima } \\
\text { (pers.) }\end{array}$ & $\begin{array}{c}\text { CCR } \\
\text { (pers.) }\end{array}$ & $\begin{array}{c}\text { CCE } \\
\text { (pers.) }\end{array}$ \\
\hline & 1955 & 7358.38 & 154585.65 & 154553.8 & 31.85 & 99775 & 20599 & 2550.14 & 943.55 \\
\hline & 2011 & 7612.63 & 325661.23 & 302560.79 & 23100.44 & 151280.4 & 40341 & 4992.25 & 1887.07 \\
\hline
\end{tabular}

3. Santa Clara del Mar: 17/23 $=0.739$.

4. Camet Norte: $3 / 23=0.13$.

5. La Caleta: $3 / 23=0.13$.

6. Mar de Cobo: $6 / 23=0.26$.

7. Balneario Parque Mar Chiquita: $11 / 23=0.478$.

$\mathrm{CG}=(0.434+0.478+0.739+$ $0.13+0.13+0.26+0.478) / 7$. $\mathrm{CG}=0.378$

Incluyendo infraestructura, equipamiento, personal y respaldo jurídico, la Capacidad de Gestión promedio es de 0.378. Entonces, si CCE $=\mathrm{CCR} \times \mathrm{CG}=>\mathrm{CCE}=4992.25 \mathrm{x}$ $0.378=>\mathrm{CCE}=1887.07$

Los resultados se presentan resumidos en el cuadro 2 para cada una de las playas y para cada una de las secuencias temporales en que se llevó a cabo el estudio de Capacidad de Carga Turística (cuadro 3). Al final se presenta una comparación entre los años extremos para la superficie total de playa del Partido de Mar Chiquita.

\section{DISCUSIÓN}

Comparando los valores obtenidos de cada playa para los dos períodos extremos, es decir, 1955 y el 2011, se obtuvieron CCT's que aumentaron, como son los casos de Santa Elena, Santa Clara del Mar, Camet Norte y Mar de Cobo, cuya situación en común radica en que en los últimos 20 años han practicado defensas costeras logrando aumentar su superficie de playa con respecto a 1955 . De esta manera, su CCT ha aumentado independientemente de la administración que el municipio ha hecho de ellas. Los mayores cambios de CCT se dan en Santa Clara del Mar y Mar de Cobo, esto relacionado con que son los barrios que más han crecido en términos urbanísticos y donde la gestión turística de las playas ha desarrollado mejoras sustanciales, 
tanto en infraestructura como en equipamiento y servicios brindados.

Por el contrario, las CCT's que disminuyeron se dieron en Playa Dorada, La Caleta y Balneario Parque Mar Chiquita. Los casos donde se hace más notable son Playa Dorada y La Caleta. El caso de Playa Dorada tiene relación directa con el tipo de defensa costera que se realizó (Seawall). Este tipo de estructura requirió que se ocupe una gran parte de la superficie de la playa con un muro de piedra, con el objeto de defender el acantilado. Esto redujo notablemente la superficie de playa con respecto a 1955. La situación de La Caleta tiene que ver con la merma en el aporte por deriva del litoral, debido a las sucesivas obstrucciones por la instalación de espigones desde el sur. Además, son playas cuya prestación de servicios básicos al turista es mínima, debido a la falta de infraestructura y porque aún son prácticamente playas vírgenes.

Si bien la CCT de las playas ha ido creciendo en relación con el aumento de espacio de playa aprovechable, la evolución de la gestión municipal con respecto al manejo del turismo en las playas del Partido de Mar Chiquita no ha aumentado a la par, dada la falta de herramientas conceptuales, económicas y físicas para solucionar o minimizar los crecientes problemas costeros. La erosión es uno de ellos. Esto relacionado con la influencia de tormentas y sudestadas que generan la pérdida de arena del sistema $y$, por ende, una disminución de la superficie de playa aprovechable.
No menos importantes son los problemas espaciales que traen consigo las concesiones balnearias. Estas tienen por objetivo ocupar el máximo espacio posible para así colocar tantas unidades de sombra como sean posibles y lograr un mayor rédito económico. Esta situación trae aparejado el inconveniente de que las concesiones privadas mantienen año a año sus $\mathrm{m}^{2}$ de playa licitados y debido a la arriba mencionada pérdida de arena por fenómenos erosivos, quien sufre la reducción de la superficie de playa es el dominio público. Dicha situación se ve reflejada en el cuadro 2, donde vemos que para el año 1955, si bien la superficie total de playa aprovechable para uso turístico es la mitad que en el 2011, prácticamente la totalidad de la playa pertenece al dominio público. Por el contrario, para el 2011, el 92\% de la playa pertenece al dominio privado. Mientras toda la playa era pública, la capacidad de carga fue mayor, y desde el momento en que algunos sectores fueron privatizados para generar servicios, los espacios públicos se redujeron y la capacidad de carga se hizo menor. Esto nos lleva a la conclusión de que el municipio debe rever la zonificación que ejerce sobre el espacio costero para, mínimamente, repensarla equitativamente en términos de dominio público-privado.

Betancourt Fernández \& Herrera Moreno (2005); Segrado \& Arroyo (2009) mencionan que este proceso de cálculo está sometido a innumerables contemplaciones subjetivas, que hacen que los resultados no siempre reflejen la 
situación adecuada. Es imprescindible tener un conocimiento minucioso sobre el área a analizar y contar con información pública que, en la mayoría de los casos, resulta de difícil acceso. Sin embargo, para Martín et al. (2012) en su propuesta para evaluar la CCT en áreas de humedales, es posible calcular la CCT a partir de factores subjetivos $\mathrm{y}$ aún en ausencia de información relacionada con el manejo.

En relación con los trabajos de Roig (2002, 2003), Betancourt Fernández y Herrera Moreno (2005), Aranguren et al. (2008) y Zacarias (2013) se procuró ir más allá del cálculo de la CCT para un solo período, proveyendo una evolución de dicho dato y brindando explicaciones de cómo y por qué los valores se han ido modificando a lo largo de los años. De esta manera, se pretende que dicha evolución permita a los entes administradores de dichas playas sacar conclusiones, con el fin de mejorar las futuras gestiones.

\section{CONCLUSIONES}

El cálculo de la evolución de la Capacidad de Carga Turística para el sector costero del Partido de Mar Chiquita arrojó un valor de 1887.07 visitantes/día para el año 2011, comparado con los 943.55 que se obtuvieron para el año 1955. Este cálculo fomenta el hecho de que es necesario contemplar una gran variedad de factores en el momento de llevar a cabo un estudio de este tipo. Pero, más allá de lo monótono y tedioso que resulte realizar un historial de este tipo aporta un sinnúmero de conclusiones de cómo ha ido mejorando o decayendo el manejo de las playas del Partido de Mar Chiquita a lo largo de los años. Esta información brinda al municipio una base de datos a partir de la cual determinar cuáles son los puntos fuertes y débiles del manejo de las playas.

De la comparación de seis períodos de mediciones concretas en las playas del Partido de Mar Chiquita se concluye que la cantidad máxima de visitantes para la superficie total de playa pública ha aumentado en 56 años, pero así también lo ha hecho la superficie total de playa. $\mathrm{Su}$ pico de capacidad no fue alcanzado en ninguno de los dos períodos extremos del presente trabajo. Por motivos relacionados con mermas en la provisión de arena por parte de las fuentes, el máximo de capacidad fue alcanzado en un período intermedio (1987).

A aquellos municipios costeros cuyos atractivos turísticos generen una afluencia desmedida, se les recomienda adquirir en sus planes de gestión y proyección turística, los parámetros aquí medidos, para un mejor desarrollo de la actividad en el futuro.

La determinación de la CCT de cada playa es el primer paso para asegurar un buen manejo del recurso. Comparando información de afluencia turística con la CCF obtenida para cada playa, y con la CCE, se puede evaluar cuáles de ellas están sobrecargadas, las que están al límite de su capacidad, las que están en su capacidad y las que están subutilizadas. Generalmente, se deben manejar los espacios tratando de no llegar a los valores máximos indicados 
en la CCE, con el objeto de mantener un margen extra de seguridad para así asegurar la sustentabilidad del recurso.

La diferencia entre la CCF, la CCR y la CCE yace en la contemplación de factores de corrección que ajustan los valores obtenidos. Estos factores responden a particularidades de cada sitio y hacen que el valor final del cálculo se asemeje lo más posible a los valores reales. En el presente estudio, se hizo referencia también a una CCF óptima, cuya diferencia con la CCF está dada por la superficie ocupada por el visitante en el sitio. Por tal motivo, y con el objeto de dimensionar la importancia de tener en cuenta que una superficie óptima para el uso sostenible de un recurso turístico (15 $\left.\mathrm{m}^{2} / \mathrm{usuario}\right)$ dista mucho de los $4 \mathrm{~m}^{2} /$ usuario que plantean los organismos turísticos internacionales para destinos estacionales.

Es necesario que el municipio del Partido de Mar Chiquita adopte entre sus planes de gestión turística en playas, una serie de medidas que apunten a difundir información concerniente al espacio costero y su dinámica natural, a fomentar una conciencia ambiental que conlleve a buenas prácticas para con el entorno, a mejorar la calidad y cantidad del equipamiento e infraestructura turística, con el fin de brindar un disfrute de mayor calidad al visitante, a repensar el accionar de las defensas costeras actuales para solucionar los problemas de erosión y a reformular la zonificación del litoral a partir no solo de los datos aquí obtenidos, sino, y aún más importante, a partir de la coherencia de percibir que todos los espacios naturales se alteran a partir de la presencia del hombre en ellos y a partir de dicha premisa, se generan límites que deben ser respetados, con el propósito de que la calidad del recurso perdure en el tiempo.

\section{BIBLIOGRAFÍA}

Aranguren, J., Moncada, J. A., Naveda, J., Rivas, D. \& Lugo, C. (2008). Evaluación de la capacidad de carga turística en la playa Conomita, Municipio Guanta, Estado de Anzoátegui. Rev. Invest., 64, 31-61.

Becerra, N. (1997). Un análisis sistémico de politicas tecnológicas. Estudio de caso: El agro pampeano argentino 1943-1990. Buenos Aires, Argentina: Ed. Centro de Estudios Avanzados, Universidad de Buenos Aires, Argentina.

Betancourt Fernández, L. \& Herrera Moreno, A. (2005). Acerca de la capacidad de carga fisica de Playa Grande, Cayo Levantado, Samaná, República Dominicana. República de Santo, Santo Domingo: Reporte Técnico del Programa EcoMar.

Cazes, G. (1992). Fondements pour une Géographie du tourisme et des loisirs. París, Francia: Ed. Bréal.

Cifuentes, M. (1992). Determinación de Capacidad Turística en Áreas protegidas. Turrialba, Costa Rica. Turrialba, Costa Rica: Ed. Centro Agronómico Tropical de Investigación y Enseñanza (CATIE).

Cifuentes, M., Mesquita, C. A., Méndez, J., Morales, M. E., Aguilar, N., Cancino, D., Gallo, M., Jolón, M., Ramírez, C., Ribeiro, N., Sandoval, E. \& Turcios, M. (1999). Capacidad de Carga Turística en las áreas de uso público del Monumento Nacional Guayabo, Costa Rica. Turrialba, Costa Rica: 
Ed. Fondo Mundial para la Naturaleza (WWF Centroamérica) y Centro Agronómico Tropical de Investigación y Enseñanza (CATIE).

Codignotto, J. (1987). Glosario geomorfológico marino. Buenos Aires, Argentina: Ed. Asociación Geológica Argentina.

Corel Photo-Paint X5 (2008). Corel Photo-Paint ${ }^{\circledR}$, Version Graphics Suite X5. Ottawa, Canada. Corel Corporation.

Echamendi Lorente, P. (2001). La capacidad de carga turística. Aspectos conceptuales y normas de aplicación. $A n$. Geogr. Univ. Complut., 2, 11-30.

García, M. C. (2011). Escenario de riesgo climático por sudestadas y tormentas en Mar del Plata y Necochea-Quequén, Provincia de Buenos Aires, Argentina. Braz. Geogr. J., 2(2), 286-304.

Google Earth (1991). Google Earth ${ }^{\circledR}$, Version 2. Boston, EE.UU.: Free Software Foundation, Inc.

Image J (2007). Image $\mathbf{J}^{\circledR}$. Santa Clara, California, EE.UU.: Sun Microsystems, Inc. Inskeep, E. (1991). Tourism Planning: An Integrated and Sustainable Development Approach. New York, EE.UU.: Ed. Van Nostrand Reinhold.

Isla, F. I. (1997). Seasonal behavior of Mar Chiquita tidal inlet in relation to adjacent beaches, Argentina. J. Coast. Res., 13(4), 1221-1232.

Martín, V. Y., Rosell, P. \& Rosake, P. (2012, abril). Propuesta Capacidad de Carga Turística en área de humedales. Ponencia presentada en IX Jornadas Nacionales de Geografía Física, Bahía Blanca, Buenos Aires, Argentina.

Mundomanz (2013). Mundomanz.com. Recuperado en julio 5, 2013. Disponible en http://www.mundomanz.com/. Organización Mundial del Turismo (OMT). (2005). Indicadores de desarrollo sostenible para los destinos turísticos: Guía práctica. Madrid, España: Ed. OMT. Roig, F. X. (2002). Análisis de la Capacidad de Carga en los espacios litorales, calas y playas, situados en áreas naturales de especial interés de la Isla de Menorca. Turismo y transformaciones urbanas en el siglo XXI. Almería, España: Ed. Fernández Gutiérrez et al. Roig, F. X. (2003). Análisis de la relación entre Capacidad de Carga Física y Capacidad de Carga Perceptual en playas naturales de la Isla de Menorca. Invest. Geogr., 31, 107-118.

Salinas, E. (2002). Ordenación, planificación y gestión de los espacios turisticos: Aspectos teórico-metodológicos. Génova, Italia: Ed. Cappaci.

Schnack, E., Fucks, E., D’Onofrio, E. \& Baldelló, G. (2007). Criterios geomorfológicos para la determinación de la línea de ribera en el litoral atlántico de la provincia de Buenos Aires. Buenos Aires: Ed. Comisión de Investigaciones Científicas en conjunto con la Autoridad del Agua.

Segrado, R. \& Arroyo, L. (2009). El método de la capacidad de carga turística aplicado a la medición de la sustentabilidad de Cozumel, México. TURyDES, 5(2), 1-26. Silva, C. P. (2002). Beach Carrying Capacity Assessment: How important is it? J. Coast. Res., 36, 190-197.

Surfer 10 (2012). Surfer ${ }^{\circledR}$ Contouring and 3D Surface Mapping for Scientists and Engineers, Version 10.7.972. Colorado, EE.UU. Golden Software, Inc.

Zacarias, D. A. (2013). Avaliação da capacidade de carga turística para gestão de praias em Moçambique: o caso da Praia do Tofo. Revista da Gestão Costeira Integrada, 13(2), 205-214. DOI: http://dx.doi.org/10.5894/rgci345 